\title{
Acute Gastroenteritis in a Pediatric Hospital in Rio de Janeiro in Pre- and Post-Rotavirus Vaccination Settings
}

\author{
Vera S. Gouvea*, Giselly S. Dias, Ericka A. Aguiar, Adriana R. Pedro, Elisa R. Fichman, \\ Evelyn S. Chinem, Sandra P. Gomes and André L.S. Domingues ${ }^{\#}$
}

Department of Virology, Institute of Microbiology, Universidade Federal do Rio de Janeiro, Rio de Janeiro, RJ, Brazil

\begin{abstract}
A 41/2-year hospital-based survey was conducted in Rio de Janeiro to determine baseline rates of gastroenteritisrelated cases, hospitalizations, and deaths; to examine the prevalence of rotavirus strains causing admissions; and to assess the immediate impact of the nationwide rotavirus immunization program launched in March 2006. From August 2002 to May 2007, 14,473 (10.4\%) of the 139,747 consultations had AGE as primary diagnosis, 491 (3.4\%) children required hospitalization and two $(0.4 \%)$ dehydrated children died. Gastroenteritis contribution to hospitalizations varied from $\sim 2.3 \%$ in 2004 and 2006 to $6.4 \%$ in 2005 , being roughly half of them rotavirus-related cases. A gradual decrease in rotavirus strain diversity was observed from 2002 to 2005 when a single G9P[8] prevailed until April 2006. Then only short profile G9P[4] and G2P[4] strains were detected. Gastroenteritis cases were distributed year-round in a trimodal pattern with major winter peaks. Local climate apparently affected the incidence of gastroenteritis: reduction in dry years (2004 and 2007) and explosive outbreaks caused by multiple agents during the heavy rainfalls and recurrent floods of the 2005-early 2006 period. Besides rotavirus, adenovirus and calicivirus were major gastroenteritis agents of these seemingly waterborne outbreaks. In conclusion, rotavirus vaccination impacted marginally, if at all, on the incidence of childhood gastroenteritis, as favorable results obtained by comparing data from the post-vaccine period to the preceding unusual 2005 year all but disappeared when comparing to previous pre-vaccination periods, and the shift towards G2P[4] rotavirus strains may be a global trend unrelated to vaccination.
\end{abstract}

Keywords: Gastroenteritis, Diarrhea, Rotavirus, Vaccine, Epidemiology, Brazil.

\section{INTRODUCTION}

Acute gastroenteritis (AGE) is a major global problem, particularly in infants and children less than 4 years of age living in developing countries [1]. Major pathogens include bacteria, enteric parasites and viruses, among which, rotavirus is considered the major cause of severe diarrhea leading to dehydration, hospital admissions, and significant medical and societal costs [2]. Precise estimates of rotavirusrelated morbidity and mortality in Brazil are not available because viral testing is not performed routinely in specimens from children with diarrhea, either hospitalized or nonhospitalized, and test results from private clinics are not included in the unified health system (DATASUS). By using generalized linear models, a time series analysis of the mortality and morbidity patterns of severe diarrhea (hospitalizations) in children $<5$ years old in Rio de Janeiro from 1995 to 1998 , showed winter seasonality and annual cycles that suggested the predominance of rotavirus as the main pathogen [3]. Those results were consistent with studies on the etiologic agents of diarrhea conducted in several parts of the country [4]. Based on public

\footnotetext{
*Address correspondence to this author at the Departmento de Virologia, Instituto de Microbiologia, CCS Bloco I, Universidade Federal do Rio de Janeiro, Av. Carlos Chagas Filho 373, Cidade Universitária, Rio de Janeiro, RJ 21941-902, Brazil; Tel: 55-21-2560-8344 \#166; Fax: 55-21-2560-2840; E-mails: Vera.gouvea@micro.ufrj.br, veragouvea44@gmail.com

"Present Address: Department of Biological Sciences, Microbiology, Universidade Federal do Triângulo Mineiro, Uberaba, MG, Brazil
}

health considerations, the decision was made to recommend rotavirus vaccine to prevent and control severe diarrhea in Brazil. The chosen vaccine was an attenuated human rotavirus (Rotarix, GSK, Belgium) extensively tested in clinical trials in several Latin American countries [5, 6]. In Brazil, this monovalent G1P[8] rotavirus vaccine was licensed and became available in private pediatric clinics in August 2005, and by March 2006, it was incorporated into the National Childhood Immunization Program of the Brazilian Public Health System (SUS) providing countrywide free vaccination to all children at 2 and 4 months of age for the recommended two-dose regimen, respectively.

The present work aimed at determining baseline rates of AGE cases, AGE-related hospitalizations and deaths in a sentinel hospital in Rio de Janeiro, to estimate the proportion of those cases caused by rotavirus infection, and to assess any immediate impact of the rotavirus immunization program on childhood diarrhea.

\section{MATERIALS AND METHODOLOGY}

The survey was conducted at the emergency of the Instituto de Pediatria e Puericultura Martagão Gesteira (IPPMG), beginning after approval by its Ethical Committee (Proc.\#08/2002) in August 2002 and ending in May 2007, when the service was closed for building repairs. The hospital is located at the university's main campus near major expressways leading from downtown Rio de Janeiro to its lowland outskirts (Baixada Fluminense). It serves the low 
socio economic population of a nearby huge conglomerate of

over 20 communities established during the last two decades. Due to lack of a computerized information system, data were gathered from medical bulletins and patient records. Parents of children admitted for rehydration therapy were invited to participate in the study providing the child's fecal specimen for viral testing.

Results on rotavirus detection and typing have been published [7]. Adenovirus was detected by an enzyme immunoassay kindly supplied by Biomanguinhos, Fiocruz, Brazil. Vital statistics and rotavirus immunization data were obtained from the Municipality of Rio de Janeiro (www.rio.rj.gov.br) and from DATASUS, Ministry of Health of Brazil (www.datasus.gov.br).

\section{RESULTS}

A total of 139,747 consultations were recorded at the emergency service during the consecutive 58-month study period: $14,473(10.4 \%)$ had AGE as primary diagnosis and $491(3.4 \%)$ of those cases were admitted for rehydration therapy (Table 1). Monthly AGE cases varied from 102 to 528 (mean 250) within the 1528 to 3066 (mean 2410) monthly visits at the service for all medical causes. Apart from the incomplete years of 2002 and 2007, the yearly contribution of AGE to hospital admissions varied from $\sim 2.3 \%$ in 2004 and 2006 to $6.4 \%$ in 2005 , resulting in an overall mean of $3.5 \%$ of all registered hospitalizations. The temporal distribution of AGE cases and AGE-related hospitalizations showed a cyclic pattern with annual peak prevalence in winter, but great year-to-year variations in the number of other peaks, their amplitude, and month of occurrence (Fig. 1). The winter peak, usually associated with rotavirus diarrhea, was present in all four complete years of study and occurred in June 2003, 2004 and 2005 and in May 2006. Secondary early autumn peaks in March, also associated with rotavirus, and thirdly late peaks in October, not associated with rotavirus, were generally present. The atypical year of 2005 showed the highest prevalence of AGE cases, with peaks that occurred throughout the year and proceeded until the mid-winter of 2006, declining afterwards to the usual low summer level. This abnormally high number of AGE cases, diagnosed at all seasons from January 2005 to July 2006, most likely reflected the occurrence of multiple outbreaks involving distinct etiological agents as discussed below.

Although the yearly numbers of AGE-related admissions were small, their winter annual peaks coincided with the

Table 1. Number of Emergency Visits, AGE Cases and AGE-Related Hospitalizations at IPPMG During the 58 Months Study Period from August 2002 to May 2007.

\begin{tabular}{|c|c|c|c|c|c|}
\hline \multirow{2}{*}{ Year } & \multicolumn{2}{|c|}{ Consultations (\%) } & \multicolumn{3}{|c|}{ Hospitalizations (\%) } \\
\hline & All Causes & AGE Cases & All Causes & AGE-Related & $\%$ of AGE Cases \\
\hline $2002 *$ & 13501 & $1068(7.9)$ & 1050 & $33(3.1)$ & 3.1 \\
\hline 2003 & 29773 & $2786(9.4)$ & 2853 & $109(3.8)$ & 4.0 \\
\hline 2004 & 28440 & $2413(8.5)$ & 2822 & $67(2.4)$ & 2.8 \\
\hline 2005 & 30978 & $4230(13.6)$ & 3185 & $203(6.4)$ & 4.8 \\
\hline 2006 & 28214 & $3266(11.6)$ & 2943 & $66(2.2)$ & 2.0 \\
\hline $2007 *$ & 8841 & $710(8.8)$ & 1100 & $13(1.2)$ & 1.8 \\
\hline Total & 139747 & $14473(10.4)$ & 13953 & $491(3.5)$ & 3.4 \\
\hline
\end{tabular}

\footnotetext{
* Only the last 5 months for 2002 and the first 5 months for 2007.
}

vaccine

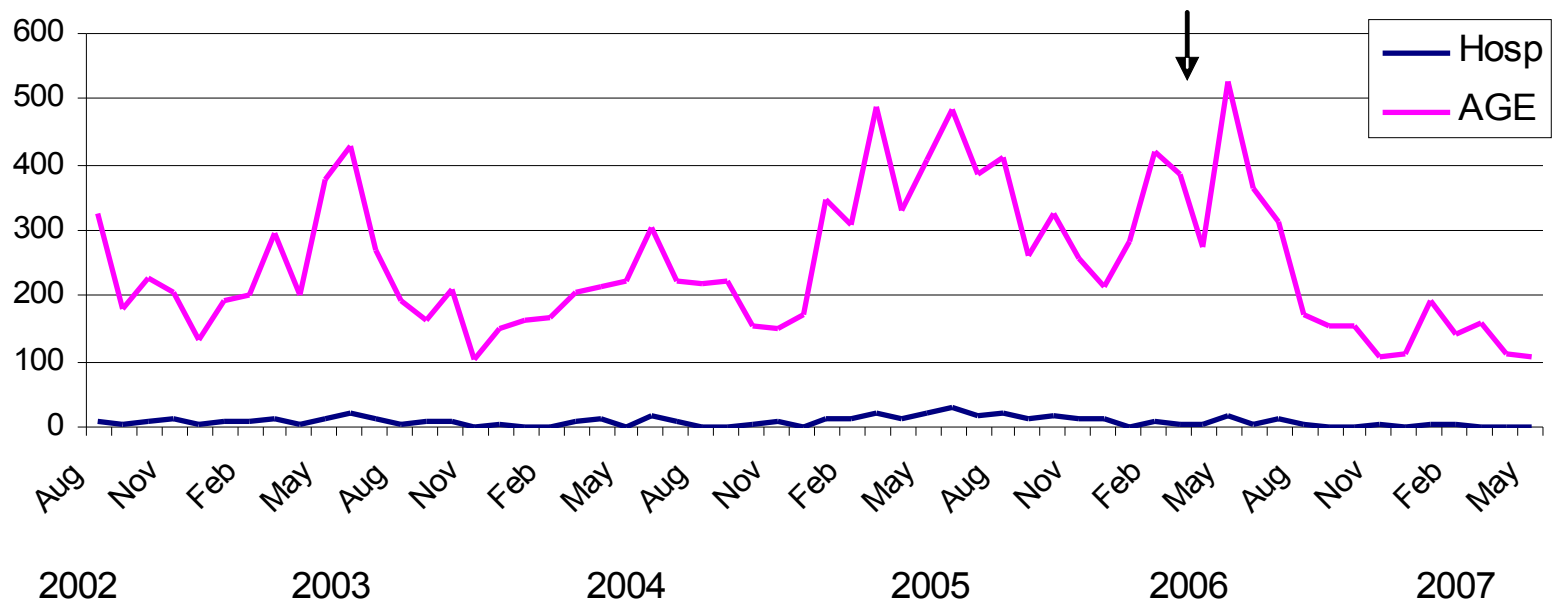

Fig. (1). AGE cases and AGE-related hospitalizations at IPPMG, Rio de Janeiro, Brazil from August 2002 to May 2007. 


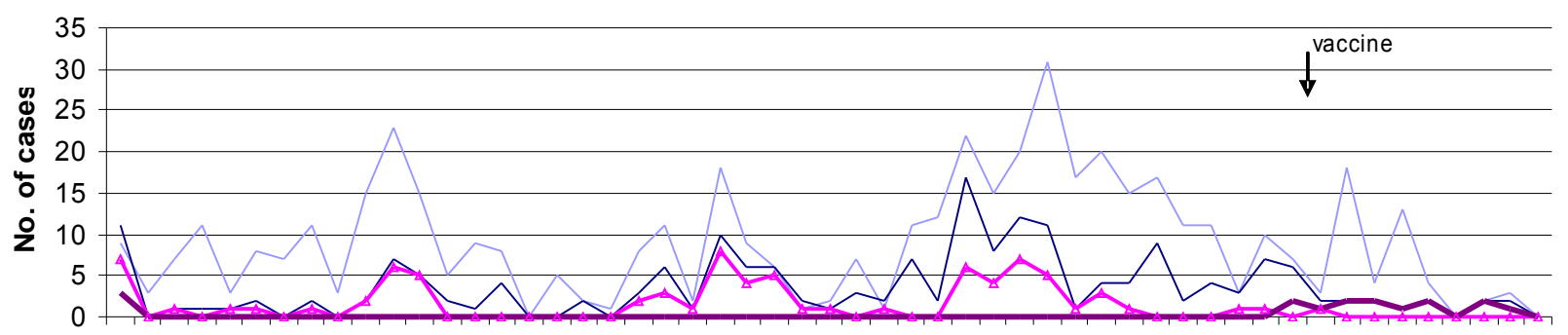

A S ONDJFMAMJ JASONDJFMAMJJASONDJFMAMJJASONDJFMAMJJASOND Month

\begin{abstract}
Admissions — Analyzed $\longleftarrow$ Rota-long —Rota-short
Fig. (2). Distribution of AGE-related and rotavirus-related hospitalizations at IPPMG from August 2002 to December 2006.
\end{abstract}

major AGE prevalence peaks (Figs. 1, 2). Overall, 193 $(\sim 40 \%)$ of the 491 hospitalized children were enrolled providing specimens for analysis. Rotavirus was found associated with $96(50 \%)$ of the severe diarrhea cases (Fig. 2). Their electropherotypes and GP genotypes were reported previously [7]. Great diversity of strains of distinct genotypes and frequent mixtures of strains were found during the first three years, with the predominant genotype gradually shifting from G1P[8] in 2002 to G9P[8] in 2004, preceding a very unusual 2005 season in which a single G9P[8] strain accounted for all the severe rotavirusassociated cases. Another change in rotavirus strains causing hospitalization was observed in April of 2006 when the common long profile, $\mathrm{P}[8]$ strains were abruptly replaced by short electropherotypes of $\mathrm{P}[4]$ specificity, mainly G9P[4] and G2P[4] strains (Fig. 2) [7]. None of the children infected with a short profile strain was eligible for rotavirus vaccination. On the other hand, one child, out of the four hospitalized children eligible for vaccination in 2006, presented severe rotavirus infection: strain G9P [8], the last long profile detected in April 2006, was recovered from this 3 -month old male child's specimen who had received the first vaccine dose two weeks before onset of illness. From January to May 2007, five vaccinated children were hospitalized due to AGE but specimens were not collected. Group $\mathrm{C}$ rotavirus was diagnosed in a single severe diarrhea case in July 2003, giving an overall $0.5 \%$ prevalence. Adenovirus was detected in a child's specimen by the end of December 2004. Afterwards, five more adenovirus-related cases were found dispersed throughout the following year, accounting for the high annual rate of $7.5 \%$ adenovirusrelated admissions and an overall $3.1 \%$ prevalence during the 58-month study period.

In general, children in the 12 to 35 -month old group accounted for the highest proportion of both AGE cases (34.4\%) and AGE-related admissions (39.1\%) at IPPMG, but the chance of requiring hospitalization due to dehydrating diarrhea was inversely proportional to the child's age, with infants less than 6-month old having about twice the chance of requiring rapid intravenous fluid replacement than older infants (Table 2). Four infants $<2$-month old were hospitalized due to AGE (in June 2003, March, April and September 2005), but none were enrolled for viral testing. The male:female rate of hospitalized children was 1.2 (54.4\% male and $45.6 \%$ female) with a slight predominance of male children hospitalized at all years except 2006 .

There were two AGE-related deaths recorded during the study period: both fatality cases were severely dehydrated 8 and 6 months old male infants admitted in January 2003 and May 2006. Specimens were not collected and details will be published elsewhere.

\section{DISCUSSION}

This single hospital-based survey spanned almost 5 years and demonstrated that a tenth of the emergency consultations had AGE as the primary diagnosis and that only a small proportion of those $(3.4 \%)$ required hospitalization. Overall, AGE contributed with $3.5 \%$ of the hospitalizations for all medical causes, and rotavirus contributed with roughly half $(1.75 \%)$ of those hospital admissions. However, the epidemiology of both AGE and rotavirus-related hospitalizations showed significant year-to-year variation. Among the factors that influence spread and transmission of

Table 2. Distribution and Prevalence of AGE-Related Cases, AGE-Related Hospitalizations, and Rotavirus-Related Admissions at IPPMG by Age Group

\begin{tabular}{|c|c|c|c|c|c|}
\hline \multirow{2}{*}{} & \multicolumn{4}{|c|}{ Age Group (Months) } \\
\cline { 2 - 6 } & $<\mathbf{6}$ & $\mathbf{6 - 1 1}$ & $\mathbf{1 2}-\mathbf{3 5}$ & $\mathbf{3 6}-\mathbf{6 0}$ & $>\mathbf{6 0}$ \\
\hline \hline AGE cases (\%) & $693(4.8)$ & $2361(16.3)$ & $4973(34.4)$ & $3429(23.7)$ & $3017(20.8)$ \\
\hline Hospitalizations (\%) & $49(10.0)$ & $101(20.6)$ & $192(39.1)$ & $84(17.1)$ & $65(13.2)$ \\
\hline \%Admission rate & 7.0 & 4.3 & 3.9 & 2.5 & 2.2 \\
\hline Rotavirus positive/analysed & $18 / 34$ & $31 / 50$ & $37 / 76$ & $7 / 24$ & $3 / 9$ \\
\hline \% Rotavirus rate* & 53 & 62 & 49 & 29 & 33 \\
\hline
\end{tabular}

*Based on laboratory analysis of $40 \%$ of the AGE-related hospitalizations [7]. 
gastroenteritis agents is local climate. Rio de Janeiro has a subtropical climate with monthly mean temperatures ranging from $17-20^{\circ} \mathrm{C}$ in the usually dry winter months (JuneAugust) to $32-35^{\circ} \mathrm{C}$ in the summer months (DecemberFebruary) with intermittent rainy periods from November to March. Unusual phenomena occurred in the city during the study period: a prolonged drought during 2004 was followed by an extremely wet year (2005) that begun with heavy rainfalls and repeated floods. Interestingly, the dry year of 2004 showed reduced AGE-related cases, though not rotavirus-related cases; whereas the extended rainy period of 2005-early 2006 coincided with an increased frequency of AGE cases during the entire period and a change in the pattern of rotavirus strains causing severe disease. The high diversity of rotavirus strains associated with hospitalizations observed up to 2004 in this and in other studies, which putatively represents the heterogeneous pool of endemic strains circulating in the community, was replaced by an unprecedented lack of diversity in rotavirus strains causing severe AGE in children in 2005. This seems typical of an epidemic outbreak caused either by the introduction of a novel rotavirus serotype in the community or spread by less common means of rotavirus transmission, such as waterborne $[8,9]$. But rotavirus G9P [8] was not new in the city: the mid-1990s genotype G9 pandemic struck Brazil in 1996 and G9P [8] strains have been detected since in many cities with fluctuating frequencies, as expected for any rotavirus serotype [10-13]. Furthermore, the atypical 2005 season showed a lower rotavirus detection rate $(34 \%)$ than those of the previous or following years (55-86\%) despite predominance of an epidemic strain: the characteristic bimodal yearly distribution of rotavirus-related cases and admissions was surmounted by peaks of non-rotavirus ones, clearly demonstrating the concurrence of outbreaks caused by other AGE agents, and further supporting a common, waterborne, source. Adenovirus, which has been implicated in $0-5 \%$ of AGE-related hospitalizations worldwide, was associated with $>7 \%$ of the 2005 admissions. The presence of caliciviruses and astroviruses in the collected specimens are currently being investigated, but preliminary results seem to indicate that norovirus (the most common human calicivirus) played a significant role in the 2005-early 2006 AGE cases in children as part of the major epidemic that swept the coastal area of Brazil in the autumn of 2005. Although affecting all age groups, it was extensively reported in the news media and perceived by many as a new rotavirus threat. Similar epidemic outbreaks occurred the following year when norovirus GII.4 was predominantly detected in the general population of Salvador, Bahia, and in young children hospitalized in Vitória, Espírito Santo [14, 15]. Although calicivirus infections have been traditionally associated to food or waterborne outbreaks of mild to moderate AGE in adults, they were relatively common $(33 \%$ prevalence) among young children with or without AGE in a study in São Paulo, Brazil, and have been increasingly implicated in severe cases requiring hospitalization of European children during periods of AGE epidemics [16, 17]. AGE outbreaks caused by caliciviruses have been well documented in cruise ships [18, 19]. As short cruises along the Brazilian coast aboard American or European ships are becoming increasingly affordable to middle-class Brazilians and astonishing popular during summer vacation and carnival since 2003 (1,000\% increase in number of passengers), yearround AGE outbreaks affecting all age groups apparently have followed the trend [20]. Thus, calicivirus should be considered a likely cause of hospitalization due to dehydration among young children in tourist resorts and major ports.

With an estimated cohort of 87,222 live births, rotavirus immunization program in the city of Rio de Janeiro $(\sim 6$ million inhabitants) achieved $43 \%$ full coverage ( 2 doses) in 2006 and $72 \%$ in 2007 . This result is above the average coverage for the state of Rio de Janeiro (38\% and 45\%) and for Brazil (39\% and 46\%) in 2006 and 2007, respectively. By taking into account those infants that received a single vaccine dose, it may be possible to assume that $76-88 \%$ of the newborns were exposed to the vaccine virus, inducing a herd immunity effect and protecting children in the community against severe disease caused by strains serologically related to the vaccine virus (G1 or $\mathrm{P}[8])$, but not against serologically unrelated strains such as G2P[4] and G9P[4] [5, 6]. This may explain the lack of detection of previously endemic strains during the first year postvaccination. Although no epidemic outbreak of G2P[4] rotavirus-related AGE has yet occurred in Brazil, the program faces the challenge of an apparent trend towards its global reemergence, as observed in countries that have not introduced a rotavirus vaccine $[21,22]$.

\section{CONCLUSION}

Similar to a study in Panama City [23], no major impact that could be definitely linked to the introduction of the rotavirus vaccine was found thus far: the significant reduction in AGE cases and hospitalizations observed in post-vaccination 2006-early 2007 period when compared to the unusual 2005 year practically disappeared when compared to those observed in the usual pre-vaccination years of 2003-2004; the April 2006 shift in rotavirus genotypes causing severe disease in children may reflect the spontaneous fluctuation in the prevalence of rotavirus genotypes seen anywhere in pre-vaccination times and may be marginally associated to the vaccination program. Nevertheless, this study demonstrated that any attempt to estimate the impact of the program needs to take into consideration local epidemiological features and natural trends of rotavirus disease, as well as environmental conditions and new behavioral tendencies, such as the "cruise ship fever" that overtook middle-class tourism worldwide resulting in the explosive global spread of norovirus GII4 strains [24].

\section{ACKNOWLEDGEMENTS}

We thank Cesar Bevilacqua and Lucia Evangelista at the Emergency Service for coordinating specimen collection, Tatiana Takayassu, Bruna Tarsiano, Mariana Costa, and all personnel at the Medical Archives, IPPMG, UFRJ, for helpful assistance with records and bulletins. Felowships were awarded to authors by CNPq or CAPES, Brasilia, Brazil. 


\section{REFERENCES}

[1] Kosek M, Bern C, Guerrant RL. The global burden of diarrhoeal disease, as estimated from studies published between 1992 and 2000. Bull World Health Organ 2003; 81: 197-204.

[2] World Health Organization. Rotavirus vaccines. Wkly Epidemiol Rec 2007; 82: 285-96.

[3] Kale PL, Hinde JP, Nobre FF. Modeling diarrhea disease in children less than 5 years old. Ann Epidemiol 2004; 14: 371-7.

[4] Pereira HG, Linhares AC, Candeias JAN, Glass RI. National laboratory surveillance of viral agents of gastroenteritis in Brazil. Bull PAHO 1993; 27: 224-33.

[5] Salinas B, Schael IP, Linhares AC, et al. Evaluation and safety, immunogenicity and efficacy of an attenuated rotavirus vaccine, RIX4414. Pediatr Infect Dis J 2005; 24: 807-16.

[6] Ruiz-Palacios GM, Pérez-Schael I, Velázquez FR, et al. Safety and efficacy of an attenuated vaccine against severe rotavirus gastroenteritis. N Engl J Med 2006; 354: 11-22.

[7] Gouvea VS, Domingues ALS, Naveca FG, Pedro AR, Bevilacqua CC. Changing epidemiology of rotavirus-related hospitalizations in Rio de Janeiro, Brazil, from 2002 to 2006. Open Virol J 2007; 1: 47-50.

[8] Rosa e Silva ML, Carvalho IP, Gouvea V. 1998-1999 Rotavirus seasons in Juiz de Fora, Minas Gerais, Brazil: detection of an unusual G3P[4] epidemic strain. J Clin Microbiol 2002; 40: 283742.

[9] Timenetsky MCST, Gouvea V, Santos N, Alge ME, Kisiellius J, Carmona RCC. Outbreak of severe gastroenteritis in adults and children associated with type G2 rotavirus. J Diarrhoeal Dis Res 1996; 14: 71-4.

[10] Domingues ALS, Silva MH, Gouvea V. Biotin-psoralen cDNA amplicons for genotyping rotavirus strains by dot hybridization assay. J Virol Methods 2007; 140: 228-31.

[11] Araujo IT, Ferreira MSR, Fialho AM, et al. Rotavirus genotypes $\mathrm{P}[4] \mathrm{G} 9, \mathrm{P}[6] \mathrm{G} 9$, and $\mathrm{P}[8] \mathrm{G} 9$ in hospitalized children with acute gastroenteritis in Rio de Janeiro, Brazil. J Clin Microbiol 2001; 39: 1999-2001.

[12] Martini IJ, Gennari GM, Martins SS, Gouvea VS, Gatti MSV. Changing distribution of human rotavirus serotypes during two epidemic outbreaks of gastroenteritis in Campinas, São Paulo, Brazil, 2003-2004: detection of G6 strains. J Clin Virol 2008; 43: 244-6.

[13] Domingues ALS, Morais ATS, Cruz RL, Moreira LP, Gouvea VS Rotavirus-associated infantile diarrhea in Uberaba, Minas Gerais, on the wake of the Brazilian vaccination program. J Clin Virol 2008; 43: 298-301.

[14] Campos GS, Moreau VH, Bandeira A, et al. Molecular detection and genetic diversity of norovirus in hospitalized young adults with gastroenteritis in Bahia, Brazil. Arch Virol 2008; 153: 1125-9.

[15] Ribeiro LR, Giuberti RSO, Barreira DMPG, et al. Hospitalization due to norovirus and genotypes of rotavirus in pediatric patients, state of Espírito Santo. Mem Inst Oswaldo Cruz 2008; 103: 201-6.

[16] Castilho JG, Munford V, Resque HR, Fagundes-Neto U, Vinjé J, Rácz ML. Genetic diversity of norovirus among children with gastroenteritis in São Paulo State, Brazil. J Clin Microbiol 2006; 44: 3947-53.

[17] Colomba C, Saporito L, Giammanco GM, et al. Norovirus and gastroenteritis in hospitalized children, Italy. Emerg Infect Dis 2007; 13: 1389-91.

[18] Ho MS, Glass RI, Monroe SS, et al. Viral gastroenteritis aboard a cruise ship. Lancet 1989; 2: 961-5.

[19] Isakbaeva ET, Widdowson MA, Beards RS, et al. Norovirus transmission on cruise ship. Emerg Infect Dis 2005; 11: 154-8.

[20] Estes MK, Prasad BVV, Atmar RL. Noroviruses everywhere: has something changed? Curr Opin Infect Dis 2006; 19: 467-74.

[21] Rahman M, Sultana R, Ahmed G, et al. Prevalence of G2P[4] and G12P[6] rotavirus, Bangladesh. Emerg Infect Dis 2007; 13:18-24.

[22] Khamrin P, Peerakome S, Tonusin S, et al. Changing pattern of rotavirus $\mathrm{G}$ genotype distribution in Chiang Mai, Thailand from 2002 to 2004: decline of G9 and reemergence of G1 and G2. J Med Virol 2007; 79: 1775-82.

[23] Guevara JN, López O, González G. Impacto de la introdución de la vacuna contra el rotavirus en la hospitalización por gastroenteritis aguda grave en el Hospital del Nino de la Ciudad de Panamá. Am J Public Health 2008; 24: 189-94.

[24] Verhoef L, Depoortere E, Boxman I, et al. Emergence of new norovirus variants on spring cruise ships and prediction of winter epidemics. Emerg Infect Dis 2008; 14: 238-43. 